\title{
Brain Shift Modeling for Use in Neurosurgery
}

\author{
Oskar Škrinjar ${ }^{1}$, Dennis Spencer ${ }^{2}$ and James Duncan ${ }^{1}$ \\ 1 Departments of Diagnostic Radiology and Electrical Engineering \\ 2 Department of Neurosurgery \\ Yale University, New Haven, CT 06520, USA
}

\begin{abstract}
Surgical navigation systems are used intraoperatively to help the surgeon to ascertain her or his position and to guide tools within the patient frame with respect to registered structures of interest in the preoperative images. However, these systems are subject to inaccuracy caused by intraoperative brain movement (brain shift) since they assume that the intracranial structures are rigid. Experiments show brain shifts of up to several millimeters, making it the cause of the dominant error in those systems. We propose a method for reducing this error based on a dynamic brain model. The initial model state is obtained from preoperative data. The brain tissue is modeled as a homogeneous linear visco-elastic material, although the model allows for setting the tissue properties locally. Gravity draws the brain downwards which in turn interacts with the skull and other surrounding structures. The simulation results are presented both for a $2 \mathrm{D}$ model (the mid-sagittal slice) and a 3D model. The results show the time evolution of the brain deformation. The complete $3 \mathrm{D}$ validation of the simulated brain deformation is a rather complicated task and is currently in progress within our laboratory, but a procedure is proposed for updating the model in time by one or more of several intraoperative measurements.
\end{abstract}

\section{Introduction}

The use of surgical navigation systems is a standard way to assist the neurosurgeon in navigating within the intraoperative environment, allowing her or him to "see" through the body and relate the position of the surgical instruments to the features in preoperative images. It is important that these systems be as precise as possible. Ideally, they should provide a $3 \mathrm{D}$ display of the neuroanatomical structures of interest and include visualization of surgical instruments within the same frame. Furthermore, if 3D acquisition systems were fast enough, brain shift would not cause an error. Thus, the ideal goal would be to sense 3D intraoperative volumetric information in real time. Since the brain deforms rather slowly even a slower acquisition rate would be acceptable. However, hardware for obtaining true 3D data is limited probably to MR or CT and is typically bulky and expensive, restricting surgical access and therefore is not currently widely used in the $\mathrm{OR}^{1}$.

${ }^{1}$ There exists a system using intraoperative MRI (see [5] and [6]) but it does not provide a complete $3 \mathrm{D}$ dataset of the head at a fast enough rate, but rather single slices at lower fields. 
Alternatively, one may improve the hardware of the current systems or develop new ones that would be fast and practical enough to work as described above. Another way is to obtain the 3D data preoperatively and then to register it to estimates of portions of 3D structure via video or other intraoperative sensors. Most of current systems use the latter approach (see [12], [13] and [14]). However, they assume that the brain and other intracranial structures are rigid and fixed relative to the skull. The brain or its structures of interest are registered to the image of the patient at the beginning of the surgery. While this can be done with a precision within $1 \mathrm{~mm}$ at the initial moment (see [13]), since the brain deforms in time, the accuracy of the system deteriorates. The median brain shift of points on the brain surface was estimated to range from $0.3 \mathrm{~mm}$ to $7.4 \mathrm{~mm}$ (see [1]). It is clear that the system based on the rigid brain assumption cannot achieve a precision better than a few millimeters at the outer structures. Since the deeper brain structures deform less than the outer ones the error is the largest at the cortical surface. The brain deforms even more after interventions, e.g. post-resections. Furthermore, the average brain shift for cases in which hematoma or tumors were removed was reported to be $9.5 \mathrm{~mm}$ and 7.9 $\mathrm{mm}$, respectively (see [2]). In such cases the error is even larger.

For the above reasons we propose a method that treats the brain as a dynamic deformable structure. The model initialization is based on preoperative data. The model deforms in time and will ultimately be guided by intraoperative information. Then the structures of interest can be displayed using the current model state rather than the rigid brain model obtained preoperatively. By doing this one reduces the error due to brain shift. Eventually the system will receive data about brain deformation intraoperatively using it to update the model. Possible sources of the deformation data are sets of points on the open brain surface obtained by stereo video imaging and 2D slices normal to the brain surface acquired by intraoperative ultrasound.

As we move in these directions, we note relevant work in soft tissue modeling related to recovering image-derived information, including: an attempt to intraoperatively reduce the brain shift error (see [2]), a paper on brain tissue modeling (see [9]), a computationally efficient tissue modeling for knee surgery (see [10]) and cardiac motion modeling (see [7] and [8]). It is also worth mentioning an algorithm for 3D finite element mesh generation (see [15]).

\section{Model}

\subsection{Brain Tissue Modeling}

The fact that the brain shift is a relatively small deformation facilitates the brain tissue modeling. A linear stress-strain relation is a good approximation for small tissue displacements. The model consists of a set of discrete interconnected nodes each representing a small part of the brain tissue. Nodes have masses depending on the size of the volume they represent and on the local tissue density. Each connection is modeled as a parallel connection of a linear spring and dashpot, known as the Kelvin solid model (see [3]). Like for the nodes, the connection 
parameters can depend on their position in the brain. The Kelvin solid model is a model for a visco-elastic material subject to slow and small deformations, which is exactly the case with brain shift. It is also a rather simple approach, which is a desirable property since the model deformation should be computed in real time, i.e. faster or at least at the speed of the brain deformation, since it must be displayed during the surgery. The constitutive relation for the Kelvin solid model is

$$
\sigma=q_{0} \epsilon+q_{1} \dot{\epsilon},
$$

where $\sigma$ is stress and $\epsilon$ strain, while $q_{0}$ and $q_{1}$ are local parameters.

If two nodes are at positions $r_{1}$ and $r_{2}$, have velocities $v_{1}$ and $v_{2}$, and are connected in the above fashion, then the force acting on the first node is

$$
\boldsymbol{f}_{\text {inner }}\left(\boldsymbol{r}_{1}, \boldsymbol{r}_{\mathbf{2}}, \boldsymbol{v}_{\mathbf{1}}, \boldsymbol{v}_{\mathbf{2}}\right)=\left[k_{s}\left(\left\|\boldsymbol{r}_{\mathbf{2}}-\boldsymbol{r}_{\mathbf{1}}\right\|-\boldsymbol{r}_{12}\right)-k_{d}\left(\boldsymbol{v}_{\mathbf{2}}-\boldsymbol{v}_{\mathbf{1}}\right) \boldsymbol{n}_{21}\right] \boldsymbol{n}_{\mathbf{2 1}},
$$

where $k_{s}$ is the stiffness coefficient, $k_{d}$ is the damping coefficient and $r_{12}$ is the rest length of the spring connecting the two nodes. In a general case they can vary from connection to connection depending on the local material properties. $\boldsymbol{n}_{21}$ is the unit vector from $\boldsymbol{r}_{1}$ to $\boldsymbol{r}_{2}$. Note that the same force acts on the other node but in the opposite direction.

While a continuum mechanics approach, implemented using finite elements, such as the one employed by our group for cardiac motion modeling (see [7] and [8]), better physically models the tissue, the discrete modeling approach, in addition to having reasonable results, is computationally faster. This is very important for our application, since the ultimate goal is to run the simulation in real time or close to real time.

\subsection{Modeling the Brain - Skull Interaction}

If a point mass is far enough away from a rigid body there is no interaction between them. When the point mass is closer than a "critical distance" to the rigid body there is an interaction, and the closer they are the greater the interaction, becoming very large if the point mass is very close to the body. Furthermore, this interaction is in the direction of the surface normal of the body. Based on the above intuitive reasoning, the following model of the interaction between a point mass and rigid body is proposed

$$
f_{\text {outer }}(r)=\left\{\begin{array}{ll}
C\left(\frac{1}{r^{2}}-\frac{1}{r_{0}^{2}}\right) n & , 0 \leq r \leq r_{0} \\
0 & , r>r_{0}
\end{array},\right.
$$

where $f$ is the force vector, $r$ is the minimal distance between the rigid body and point mass, $r_{0}$ is the "critical distance", $C$ is a proportionality constant and $n$ is the body surface normal. This force acts on both the rigid body and point mass but in the opposite directions.

If a brain model node comes closer than $r_{0}$ to the skull it interacts with the skull in the above way. In small deformation situations, such as the brain shift case, only the outer brain surface nodes will interact with the skull, which reduces the simulation time. Since the skull is assumed to be fixed, the skull brain interaction in the simulation is applied only to the brain nodes. 


\subsection{The Model Equations}

Newton's Second Law for node $j$ gives

$$
m^{j} \boldsymbol{a}^{j}=m^{j} \boldsymbol{g}+\boldsymbol{f}_{\text {outer }}^{j}+\sum_{i=1}^{n^{j}} f_{\text {inner }}^{j}{ }_{s_{i}^{j}}
$$

where $m^{j}$ is the node's mass, $\boldsymbol{a}^{j}$ is its acceleration, $\boldsymbol{f}_{\text {outer }}{ }^{j}$ is its interaction with the skull defined by $(3), f_{i n n e r}{ }_{s_{i}^{j}}^{j}$ is the interaction between nodes $j$ and $s_{i}^{j}$ defined by (2) and $g$ is the gravity acceleration, while $\left\{s_{1}^{j}, s_{2}^{j}, \ldots, s_{n^{j}}^{j}\right\}$ is the set of the neighboring nodes of the node $j$. Equation (4) represents a system of second order nonlinear ordinary differential equations.

One can define the state variables to be $x_{2 j-1}=r^{j}$ and $x_{2 j}=v^{j}$ for $j=$ $1, \ldots, N$, where $N$ is the number of the brain model nodes. Obviously, $\dot{x}_{2 j-1}=$ $x_{2 j}$. The expression for $\dot{x}_{2 j}$ follows directly from (4). It depends only on state variables but not on their time derivatives. Now it is clear that (4) can be rewritten in the compact state-space form

$$
\dot{\mathcal{X}}=f(\mathcal{X})
$$

where $\mathcal{X}$ is the vector of the state variables. It is assumed that the brain starts deforming from a rest position, i.e. $v^{j}(t=0)=0$ for all $j$. The initial node positions $r^{j}(t=0)$ were obtained from the preoperative images, as discussed in the next section.

The system defined by (5) is suitable for numerical integration (see [4]). In this case the fourth order Runge-Kutta method with adaptive stepsize was employed.

\section{Results}

\subsection{D Results}

The mid-sagittal slice of a MRI data set is chosen for testing the $2 \mathrm{D}$ model. We developed a tool in MATLAB for preprocessing the slice and setting the initial model state. A part of the skull is artificially erased to simulate the craniotomy. The tool allows one to set the tissue properties, which affect the node masses and connection parameters, to define the initial node positions as well as the orientation of the head. It also offers an initial level of automation. Figure 1 shows the head in a certain orientation and the corresponding $2 \mathrm{D}$ mesh.

As the model deforms in time, the nodes change positions and the image of the brain deforms. Since the deformation is rather small one can assume that the interior of each triangle in the model deforms in an affine manner, as dictated by the following equations. Let $\left(x_{1}^{i}, y_{1}^{i}\right),\left(x_{2}^{i}, y_{2}^{i}\right)$ and $\left(x_{3}^{i}, y_{3}^{i}\right)$ be the node coordinates of a triangle in the initial state and $\left(x_{1}^{c}, y_{1}^{c}\right),\left(x_{2}^{c}, y_{2}^{c}\right)$ and $\left(x_{3}^{c}, y_{3}^{c}\right)$ 

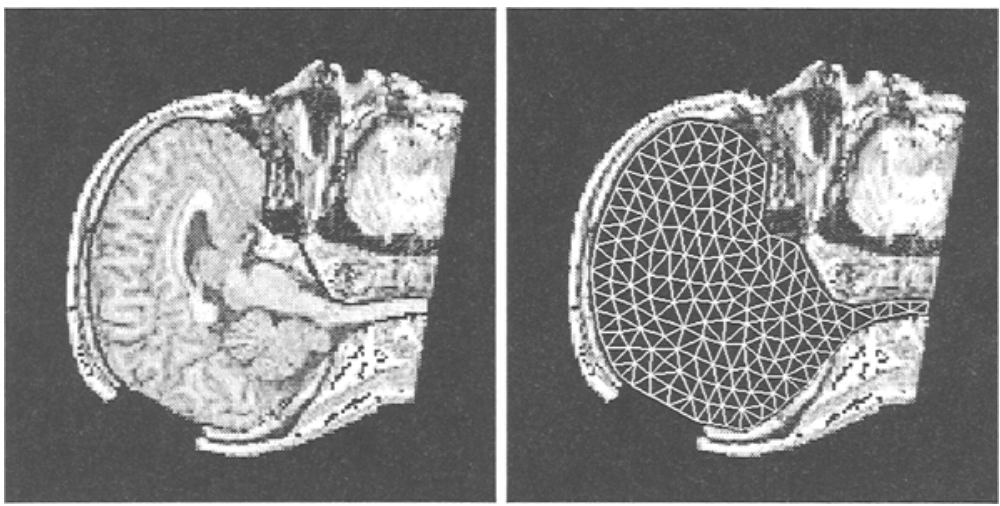

Fig. 1. The initial node positions as well as the connection properties are set by a special purpose tool developed in MATLAB.

their corresponding coordinates in the current state. One can define local triangle coordinates $\alpha$ and $\beta$ through the following equation

$$
\left[\begin{array}{l}
x^{i} \\
y^{i}
\end{array}\right]=\left[\begin{array}{l}
x_{1}^{i} \\
y_{1}^{i}
\end{array}\right]+\alpha\left[\begin{array}{l}
x_{2}^{i}-x_{1}^{i} \\
y_{2}^{i}-y_{1}^{i}
\end{array}\right]+\beta\left[\begin{array}{l}
x_{3}^{i}-x_{1}^{i} \\
y_{3}^{i}-y_{1}^{i}
\end{array}\right]
$$

where $0 \leq \alpha \leq 1,0 \leq \beta \leq 1-\alpha$ and $\left(x^{i}, y^{i}\right)$ is a point inside or at the boundary of the initial triangle. The affine transformation is defined by

$$
\left[\begin{array}{l}
x^{c} \\
y^{c}
\end{array}\right]=\left[\begin{array}{l}
x_{1}^{c} \\
y_{1}^{c}
\end{array}\right]+\alpha\left[\begin{array}{l}
x_{2}^{c}-x_{1}^{c} \\
y_{2}^{c}-y_{1}^{c}
\end{array}\right]+\beta\left[\begin{array}{l}
x_{3}^{c}-x_{1}^{c} \\
y_{3}^{c}-y_{1}^{c}
\end{array}\right]
$$

where $\left(x^{c}, y^{c}\right)$ is the point in the current triangle corresponding to $\left(x^{i}, y^{i}\right)$. One can eliminate $\alpha$ and $\beta$ from (6) and (7) obtaining the direct affine transformation between the two triangles. The transformation is invertable as long as both triangles have non-zero areas. This is true since the deformation is small and triangles do not change shape significantly, preserving non-zero areas. Equations (6) and (7) are used to calculate the image deformation knowing the node positions in time.

Although the tissue properties could be set locally, at this point the brain is assumed to be homogeneous. A time sequence of a deforming brain is shown in Fig. 2. Figures 3 and 4 show the difference in the brain deformation if the tissue is made stiffer ${ }^{2}$, but still homogeneous. The final (rest) state is the state after which there is no noticeable change in the model. For a $2 \mathrm{D}$ brain model with 176 brain nodes, 57 skull (rigid) nodes and 469 connections it takes approximately ten minutes to simulate the brain deformation to the rest state on a Hewlett Packard 9000 (C110) machine. However, the code was not optimized for an

${ }^{2}$ The "stiffer" case has 10 times greater the stiffness coefficient $\left(k_{s}\right.$ in $\left.(2)\right)$ than the "softer case". 
efficient computation. The primary goal was to test the model but certainly there are many ways to go about reducing the simulation time. More simulation results can be found at http://pantheon.yale.edu/ os $28 /$.
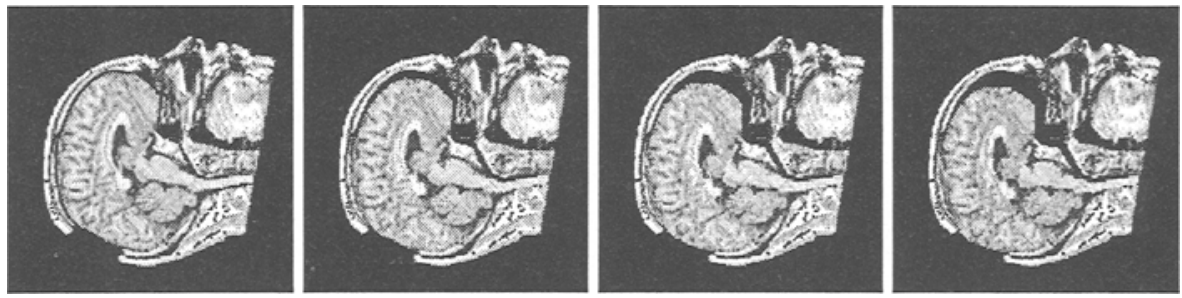

Fig. 2. A time sequence showing the brain model deformation. From left to right are the initial state, two middle states and the final (rest) state after equal intervals. As the brain settles down due to gravity, there are not only posterior movements, but also slight extensions in superior and inferior directions.

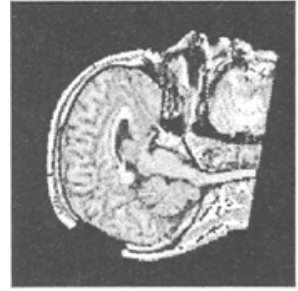

(a)

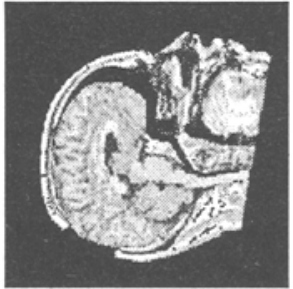

(b)

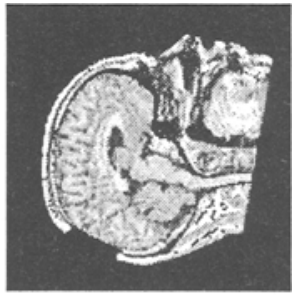

(c)

Fig. 3. Deformation comparison of the models with different tissue properties. (a) initial state, (b) final (rest) state for a "softer" case, (c) final (rest) state for a "stiffer" case. Note the difference in the curvature of the brain boundary at the position of the skull opening for the two cases. The largest deformation is at the anterior part of the brain.

\subsection{D Results}

The initial state geometry of the 3D model is based on a MRI dataset of a patient's head. The aforementioned tool allows one to create an artificial craniotomy, set tissue properties and the model orientation and automatically set the model connections and initial node positions. The equations driving the model are completely analogous to the ones in the $2 \mathrm{D}$ case, the only difference being the number of nodes and connections. Figure 5 represents a time sequence of 


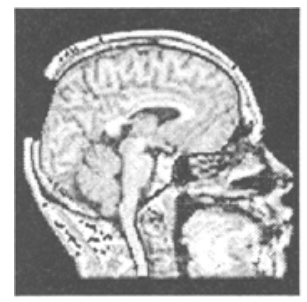

(a)

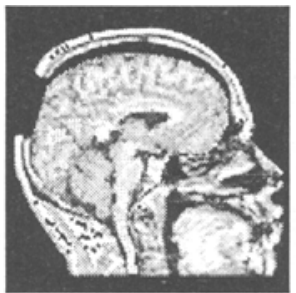

(b)

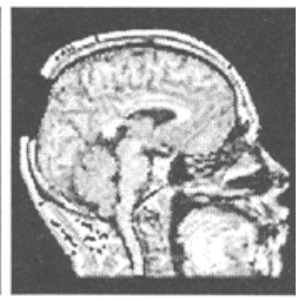

(c)

Fig. 4. Deformation comparison of the models with different tissue properties (another angle). (a) Initial state, (b) final (rest) state for a "softer" case, (c) final (rest) state for a "stiffer" case. Note the deformation at the top of the superior part of the brain.

states of the model in a certain orientation. The sequence shows how the brain settles down, supported by the skull and other surrounding structures. Figure 6 shows a time sequence of states for the model in another orientation.

The 3D model has 1093 nodes and 4855 connections. It takes approximately four hours for the simulation to reach the final state. At the other hand a real brain typically deforms for 30 minutes, which is about 8 times smaller than the simulation time. However, we believe that computational time can be reduced to or close to 30 minutes for the following reasons. We employed the fourth order Runge-Kutta method for numerical integration which is not the fastest method (see [4]). Also, we imposed rather strict conditions on the precision (required for the adaptive stepsize control part). By relaxing them one can increase the computational efficiency. Moreover, one can optimize the data organization for the specific application further reducing the simulation time. Finally, the computational time can be reduced by using a faster computer.
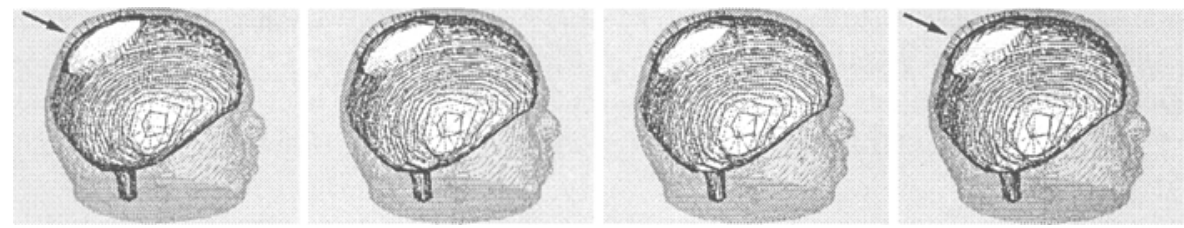

Fig. 5. A time sequence of model states equidistant in time. The left image represents the initial state while the right one shows the final (rest) model state. The brain slightly settles down due to gravity. One can notice through the artificially made craniotomy the increase in the gap between the skull and the brain over time (the black gap under the arrow). For larger images refer to http://pantheon.yale.edu/ os $28 /$. 

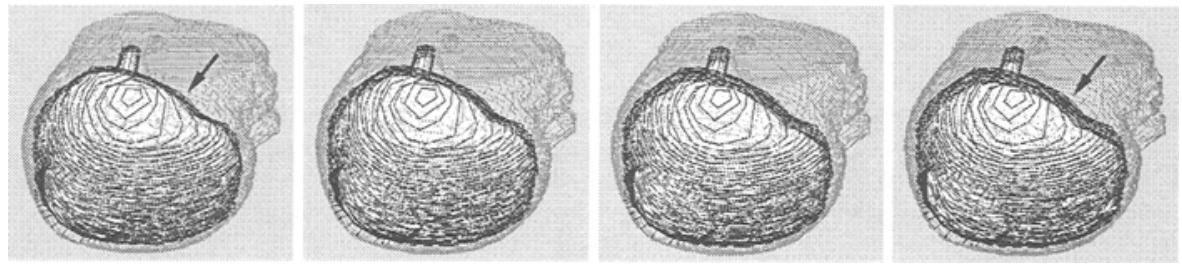

Fig. 6. Another time sequence of model states equidistant in time. The left image represents the initial state while the right one shows the final (rest) model state. Note how the brain settles down (the increase in the top black gap under the arrow). As opposed to Fig. 5 in this case the gap between the brain and the skull at the craniotomy decreases over time.

\section{Discussion}

The two main problems with this modeling are how to reliably estimate the model parameters and how to validate the model deformation. Research is being conducted in our laboratory addressing both problems. Since the physical brain tissue properties in humans and animals are very similar one can perform tissue property measurements on an animal brain. Those results can be used to initialize the model parameters. However, brain tissue properties might change during the surgery due to reducing intracranial pressure, by controlling $\mathrm{CO}_{2}$ concentration in the blood and reducing the water content of the brain by administering an osmotically active drug. Cerebrospinal fluid also leaks from the subarachnoid space at a variable rate. We are working on including these effects both directly in the model as well as through intraoperative information that will guide the model. Currently our model incorporates the visco-elastic brain tissue properties, initially set homogeneously within the brain, and the effect of gravity. In addition, our current research includes cerebrospinal fluid modeling motivated by interaction between fluid particles given by Lennard-Jones model (see [11], Chapter 15). Since the grey and white matter do not have the same visco-elastic properties the geometry of the sulci affects the brain deformation. This is also a problem we are working on.

We believe that our model, with appropriately set tissue parameters and intraoperatively guided, can significantly reduce the error introduced by the brain shift, making the surgical navigation system more precise and reliable.

\section{Acknowledgements}

We are grateful to prof. Turan Onat from Department of Mechanical Engineering, Yale University, for useful discussions as well as to graduate students Sudhakar Chelikani and Aleksandar Milošević from the Departments of Applied Physics and Physics, Yale University, respectively. 


\section{References}

1. Hill, D., Maurer, C., Wang, M., Maciunas, R., Barwise, J., Fitzpatrick, M.: Estimation of Intraoperative Brain Surface Movement. CVRMed-MRCAS'97, Grenoble, France, March (1997) 449-458

2. Bucholz, R., Yeh, D., Trobaugh, J., McDurmont, L., Sturm, C., Baumann, C., Henderson, J., Levy, A., Kessman, P.: The Correction of Stereotactic Inaccuracy Caused by Brain Shift Using an Intraoperative Ultrasound Device. CVRMed-MRCAS'97, Grenoble, France, March (1997) 459-466

3. Pamidi, M. R., Advani, S. H.: Nonlinear Constitutive Relations for Human Brain Tissue. Journal of Bio-Mechanical Engineering, February (1978), Vol. 100, 44-48

4. Press, W. H., Teukolsky, S. A., Vettrling, W. T., Flannery, B. P.: Numerical Recipes in $C, 2^{\text {nd }}$ Edition, Camridge University Press, 1992.

5. Ferenc, A. J.: Image-guided Procedures and the Operating Room of the Future. Radiology, September (1997), Volume 204, 601-612

6. Revolution in the O.R., Brigham and Women's Hospital, $B W H$ Fall 1997, 10-16

7. Shi, P., Sinusas, A. J., Constable, R. T., Duncan, J. S.: Volumetric Deformation Analysis Using Mechanics-Based Data Fusion: Applications in Cardiac Motion Recovery. International Journal of Computer Vision, Kluwer, submitted.

8. Duncan, J., Shi, P., Constable, R.T., Sinusas, A.: Physical and Geometrical Modeling for Image-Based Recovery of Left Ventricular Deformation. Progress in Biophysics and Molecular Biology, in press.

9. Edwards, P. J., Hill D. L. G., Little, J. A., Hawkes, D. J.: Deformation for Image Guided Interventions Using a Three Component Tissue Model. 15th International Conference, IPMI'97, Proceedings, (1997) 218-231

10. Gibson, S., Samosky, J., Mor, A., Fyock, C., Grimson, E., Kanade, T., Kikinis, R., Lauer, H, McKenzie, N., Nakajima, S., Ohkami, H., Osborne, R. Sawada, A.: Simulating Arthroscopic Knee Surgery Using Volumetric Object Representations, Real-Time Volume Rendering and Haptic Feedback. CVRMed-MRCAS'97, Grenoble, France, March (1997) 369-378

11. Fosdick, L. D., Jessup, E. R., Schauble, C. J. C., Domik, G.: An Introduction to High-Performance Scientific Computing, The MIT Press, 1995.

12. Grimson, W. E. L., Ettinger, G. J., White, S. J., Gleason, P. L., Lozano-Perez, T., Wells III, W. M., Kikinis, R.: Evaluating and Validating an Automated Registration System for Enhanced Reality Visualization in Surgery.

13. Grimson, W. E. L., Ettinger, G. J., White, S. J., Gleason, P. L., Lozano-Perez, T., Wells III, W. M., Kikinis, R.: An Automatic Registration Method for Frameless Stereotaxy, Image Guided Surgery, and Enhanced Reality Visualization. IEEE Transactions on Medical Imaging, Vol. 15, No. 2, April (1996) 129-140

14. Peters, T., Davey, B., Munger, P., Comeau, R., Evans, A., Olivier, A.: ThreeDimensional Multimodal Image-Guidance for Neurosurgery. IEEE Transactions on Medical Imaging, Vol. 15, No. 2, April (1996), 121-128

15. Hartmann U., Kruggel F.: A Fast Algorithm for Generating Large Tetrahedral 3D Finite Element Meshes from Magnetic Resonance Tomograms. Workshop on Biomedical Image Analysis, Proceedings, Santa Barbara, CA, June (1998) 184-191 